\title{
Safety and efficacy of COVID-19 vaccines in pregnant women with rheumatic diseases: an immunologic perspective
}

\author{
Javeria Tariq $^{1}$ (D) Latika Gupta ${ }^{2}$ (D)
}

Received: 2 June 2021 / Accepted: 5 June 2021 / Published online: 10 June 2021

(c) The Author(s), under exclusive licence to Springer-Verlag GmbH Germany, part of Springer Nature 2021

Vaccine hesitancy is widely prevalent, and, notably, despite best efforts, no country has yet exceeded complete vaccination of more than $60 \%$ of their population. Testing times, such as a raging pandemic call for triage and gathering evidence suited to the masses, while minorities of any kind (social, ethnic, racial, and scientific) inevitably stand ignored. While global vaccination has now begun, it is imperative to focus on understanding efficacy and safety amongst the pregnant and lactating women while actively nipping rumors in the bud.

The safety of vaccine in Rheumatic disease (RD) patients is unclear, and the safety in pregnant women with RD is even more critical to know since neither of these groups have been included in the clinical trials. Among pregnant women, safety of the mother and the fetus, and efficacy have emerged as the prime concerns [1]. Pregnancy is a state where the immune cells involved in antibody production may respond differently and thus affect the efficacy of a vaccine negatively. But this concept of immune modulation during pregnancy requires a deeper understanding of maternal immune response to the specific vaccine considering influenza vaccine has no difference in efficacy when compared to nonpregnant groups [2].

The risk of flare of symptoms and other potential side effects particularly in those with autoimmune conditions is another widely expressed concern. Vaccination among lactating women is another gray area, with recommendations largely based on understanding of vaccine and antibody

Latika Gupta

drlatikagupta@gmail.com

Javeria Tariq

javeria.tariq@scholar.aku.edu

1 Medical College, The Aga Khan University, National Stadium Road, Karachi 74800, Pakistan

2 Department of Clinical Immunology and Rheumatology, Sanjay Gandhi Postgraduate Institute of Medical Sciences, Raebareli Road, Lucknow 226014, India pharmacodynamics. The Covaxin and Sinovac vaccines contain immune activators, such as phosphate-buffered saline and Imidazoquinolinone (TLR Agonist), and hence which could be responsible for flaring of the underlying autoimmune disease like Rheumatoid arthritis [3]. For the patients with rheumatic diseases, the theoretical risk for relapse of the autoimmune disease is related to mRNA's properties. By RNA sensor recognition in dendritic cells, mRNA from the vaccines can exert adjuvant-life effects on the innate immune system [4]. However, the Korean College of Rheumatology issued a set of guidelines for patients with Autoimmune Inflammatory Rheumatic Diseases encouraging the use of vaccines after consultancy with their rheumatologists [5]. A study by Geisen et al. further supported the safety of mRNA vaccine in immunosuppressed patients with chronic inflammatory conditions [6] but the safety in pregnant population with such conditions taking immunosuppressive regimens, is still unclear.

A large study by Shimabukuro et al. recently described no glaring concerns except spontaneous abortions in pregnant women who got the mRNA vaccine [7]. It was proposed that antibodies against SARS-Cov2 spike protein might cross-react with human placental protein syncytin 1 , causing damage to placenta, but no similarity was noticed in amino acid sequence of both proteins. Notably, rates of miscarriage were numerically lower in accidental pregnancies among the vaccinated in the UK [8], further supporting its safety during pregnancy. However, amid emerging public advisory on the safety of mRNA vaccine based on the aforementioned cohort data, Professor Hooman has raised valid concerns on the poor understanding of safety in selected subgroups of pregnant women. Emerging reports of severe immune reaction in those who get vaccinated while harboring an active COVID-19 infection may indeed be of concern. Similarly, the effect of maternally transferred antibodies in a breast-feeding neonate who contracts COVID-19 is largely unknown. Anaphylaxis and TTP are other rare concerns with vaccination, with specific risks in the pregnant and lactating 
population being unclear [9]. It is imperative for stakeholders to join hands to explore outcomes in these scenarios, to avoid public outcry from unexpected immune faux pas. It has been hypothesized that vaccine-induced immunity is better when compared to immunity after contracting SARS$\mathrm{CoV}-2$ infection and thus vaccination is seen as the ultimate goal to curb the pandemic [10].

Notably, hue and cry from anti-Vaxxers can gain foothold in scientific publications through secondary citations an issue that scientists and administrators may be made aware of [11]. Counseling patients regarding the passive immunity for the newborn and the benefits in long-run is not possible without a scientific proof. Until we get evidence to encourage immunization in pregnant women, utilization of guidelines like those being practiced in Japan can prove effective, whereby pregnant population is provided the vaccine on basis of informed consent, with priority being given to those who work as front-line workers or have underlying co-morbid like diabetes or hypertension. The doses are avoided during first trimester to avoid any harm to development of fetal organs [12]. Meanwhile, family members of the pregnant population should be vaccinated, and the exposure should be reduced as far as possible. Other basic SOPs should be strictly followed to avoid contracting COVID-19 which is known to present more intensely in pregnant women, considering an increased susceptibility to any disease.

There has been hysteria associated with risk to fetus from COVID-19 vaccine administered to pregnant women; however, it is poorly understood by the population that the best way neonate can be protected is by placental transfer of antibodies to the fetus [13]. A recent case of normal delivery in a lady vaccinated for COVID-19 during third trimester showed presence of antibodies in the baby [14]. Besides, immunization for influenza and pertussis during pregnancy has been in practice for decades and has proved vital to prevent these infections in neonate [15]; hence, a similar trend may be expected with COVID-19 vaccines.

Another area of confusion is the development of significant antibody titers in RD patients under immunosuppressive treatment, with serious lacunae in the understanding of the immune events in pregnant women [3]. Recent studies show promising effects of antibody development in pregnant women after mRNA vaccination which crosses the placenta and protects the fetus. Also, placental examination of vaccinated women was no different than those of non-vaccinated reducing concerns of antibody-induced placental damage $[16,17,18]$. This opens the doors to confirm immunogenicity in pregnant women with RDs.

The pyramid of evidence approach is crumbling under pressure of the raging pandemic. While emerging openaccess collaborative data sets are now in vogue as federal agencies clamor for information, there is a need to strengthen approaches to collect data for post-vaccine surveillance [19].
Countries with nationalized registries may be appropriately placed to lead such initiative, with a greater focus on educating lay public to diligently report adverse effects and avoid misinformation being vital.

All previous global pandemics have been dealt with emergence of vaccines and achieving herd immunity. Hence, the vaccines should be made available to pregnant patients as recommended by ACOG; however, they should be given the freedom to decide with the help of their obstetrician on the basis of risk-to-benefit ratio, keeping in mind lack of data available and a potential of harm to fetus, while also a risk of ADE if previously infected with SARS-CoV2 or contracting COVID-19 infection if not vaccinated which itself is associated with a high morbidity and mortality in pregnancy. Pregnant women who choose to get vaccinated should be encouraged to self-report any effects by contacting their primary caregivers and online registries can be made for this to better deal and understand with issues, thus helping to curb the prevailing misinformation.

Authors' contribution Conceptualization LG. Investigation/data curation JT, LG. Writing original draft JT, LG. Review and editing LG.

Funding This study was not funded.

\section{References:}

1. GoncuAyhan S, Oluklu D, Atalay A et al (2021) COVID-19 vaccine acceptance in pregnant women. Int J Gynecol Obstet. https:// doi.org/10.1002/ijgo.13713

2. Saeed Z, Greer O, Shah NM (2020) Is the host viral response and the immunogenicity of vaccines altered in pregnancy? Antibodies 9:38. https://doi.org/10.3390/antib9030038

3. Schulze-Koops H, Specker C, Skapenko A (2021) Vaccination of patients with inflammatory rheumatic diseases against SARSCoV-2: considerations before widespread availability of the vaccines. RMD Open. https://doi.org/10.1136/rmdopen-2020-001553

4. Velikova T (2021) Georgiev T (2021) SARS-CoV-2 vaccines and autoimmune diseases amidst the COVID-19 crisis. Rheumatol Int 413(41):509-518. https://doi.org/10.1007/S00296-021-04792-9

5. Park JK, Lee EB, Shin K et al (2021) COVID-19 vaccination in patients with autoimmune inflammatory rheumatic diseases: clinical guidance of the Korean College of Rheumatology. J Korean Med Sci 36:1-12. https://doi.org/10.3346/jkms.2021.36.e95

6. Geisen UM, Berner DK, Tran F et al (2021) Immunogenicity and safety of anti-SARS-CoV-2 mRNA vaccines in patients with chronic inflammatory conditions and immunosuppressive therapy in a monocentric cohort. Ann Rheum Dis. https://doi.org/10.1136/ annrheumdis-2021-220272

7. Shimabukuro TT, Kim SY, Myers TR et al (2021) Preliminary findings of mRNA Covid-19 vaccine safety in pregnant persons. N Engl J Med. https://doi.org/10.1056/NEJMoa2104983

8. Male V (2021) Are COVID-19 vaccines safe in pregnancy? Nat Rev Immunol 21:200-201

9. Garnier M, Curado A, Billoir P, Barbay V, Demeyere M, Dacher J-N (2021) Imaging of Oxford/AstraZeneca ${ }^{\circledR}$ COVID-19 
vaccine-induced immune thrombotic thrombocytopenia. Diagn Interv Imaging. https://doi.org/10.1016/j.diii.2021.04.005

10. Velikova $T$ (2021) Infection-acquired versus vaccine-induced immunity against COVID-19. Cent Asian J Med Hypotheses Ethics 2:29-35

11. Khan H, Gasparyan AY, Gupta L (2021) Lessons learned from publicizing and retracting an erroneous hypothesis on the mumps, measles, rubella (MMR) vaccination with unethical implications. J Korean Med Sci. https://doi.org/10.3346/jkms.2021.36.e126

12. Hayakawa S, Komine-Aizawa S, Takada K et al (2021) AntiSARS-CoV-2 vaccination strategy for pregnant women in Japan. J Obstet Gynaecol Res. https://doi.org/10.1111/jog.14748

13. Craig AM, Hughes BL, Swamy GK (2021) Coronavirus disease 2019 vaccines in pregnancy. Am J Obstet Gynecol 3:100295

14. Gill L, Jones CW (2021) Severe Acute Respiratory Syndrome Coronavirus 2 (SARS-CoV-2) antibodies in neonatal cord blood after vaccination in pregnancy. Obstet Gynecol 137:894-896. https://doi.org/10.1097/AOG.0000000000004367

15. Adhikari EH, Spong CY (2021) COVID-19 vaccination in pregnant and lactating women. JAMA 325:1039. https://doi.org/10. 1001/jama.2021.1658

16. Collier A-RY, McMahan K, Yu J et al (2021) Immunogenicity of COVID-19 mRNA vaccines in pregnant and lactating women. JAMA. https://doi.org/10.1001/jama.2021.7563
17. Beharier O, Plitman Mayo R, Raz T et al (2021) Efficient maternal to neonatal transfer of antibodies against SARS-CoV-2 and BNT162b2 mRNA COVID-19 vaccine. J Clin Invest. https://doi. org/10.1172/JCI150319

18. Egerup P, Fich Olsen L, Christiansen AH, Westergaard D, Severinsen ER, Hviid KVR, Kolte AM, Boje AD, Bertelsen MMF, Prætorius L, Zedeler A, Nielsen JR, Bang D, Berntsen S, Ethelberg-Findsen J, Storm DM, Bello-Rodríguez J, Ingham A, OlléLópez J, Hoffmann ER, Wilken-Jensen C, Krebs L, Jørgensen FS, Westh H, Jørgensen HL, la Cour Freiesleben N, Nielsen HS (2021) Severe Acute Respiratory Syndrome Coronavirus 2 (SARS-CoV-2) antibodies at delivery in women, partners, and newborns. Obstet Gynecol 137:49-55. https://doi.org/10.1097/ AOG.0000000000004199

19. Kharbanda R, Naveen R, Misra D et al (2020) Combined case record forms for collating obstetric outcomes in rare rheumatic diseases. Indian J Rheumatol 15:191. https://doi.org/10.4103/injr. injr_102_20

Publisher's Note Springer Nature remains neutral with regard to jurisdictional claims in published maps and institutional affiliations. 